are represented. This limiting value of 4 is less than might have been expected, and this fact suggests that the contribution of the side-chains to any re. flexion is small, probably due to their large thermal motion.

In addition, the theory predicts (as can also be shown by a simpler approach) that meridional reflexions can occur only on layer lines which involve Bessel functions of order zero ; that is, at reciprocal spacings of multiples of $1 / 1 \cdot 5 \mathbf{A}^{-1}$. This had previously been pointed out by Perutz ${ }^{3}$ when reporting the strong meridional $1 \cdot 5-\mathrm{A}$. refiexion.

We have therefore no doubt that the structure of poly- $\gamma$-methyl-L-glutamate is based on a helix of eighteen residues in five turns and $27 \mathrm{~A}$., or a helix which approximates to this very closely. As the structure proposed by Pauling and Corey ${ }^{2}$ satisfies these conditions and is also stereochemically very satisfactory, it seems to us highly probable that it is correct.

We should like to thank Dr. Bamford and his colleagues for allowing us to quote their experimental results in advance of publication, and Sir Lawrence Bragg and Dr. M. Perutz for the stimulus which their interest in this work has provided.

Crystallographic Laboratory,

W. Cochran

Cavendish Laboratory, Cambridge.

Medical Research Council Unit for the Study of the Molecular Structure of Biological Systems, Cavendish Laboratory, Cambridge. Dec. 14 .

${ }^{1}$ Cochran, W., Crick, F. H. C., and Vand, V. (to be published). 2 Pauling, L., and Corey, R. B., Proc. U.S. Nat. Acad. Sci., 37, 241 (1951).

3 Perutz, M. F., Nature, 167, 1053 (1951).

:Bamford, C. YI., Brown, L., Elliott, A., Hanby, W. E., and Trotte r, I. F. (to be published).

\section{Determination of the Atomic Mass of Phosphorus by a Nuclear Reaction Method}

The $Q$-value of the reaction ${ }^{31} \mathrm{P}(d, n)^{32} \mathrm{~S}$ has been determined by the photographic plate method and used to calculate the mass of phosphorus-31. This reaction is suitable for the purpose, since an accurate redetermination of the mass of sulphur-32 (31.982218 \pm 0.000025 m.u.) has been recently reported by $\mathrm{Nier}^{1}$ using a double-focusing massspectrometer.

A thin $(60-\mathrm{keV}$.) phosphorus target deposited on a thick gold backing was bombarded by deuterons of incident energy $8 \cdot 13 \pm 0.05 \mathrm{MeV}$. The neutrons emitted wore detected by using photographic emulsion $600 \mu$ thick. From measurements of the ranges and orientations of the recoil protons in plates exposed at two angles $\left(0^{\circ}\right.$ and $\left.40^{\circ}\right)$ of emission, the energy spectra of the emitted neutrons were obtained. The $Q$-values calculated for the long-range neutron group at the two angles of observation agree well, and give $Q=6.81 \pm 0.08 \mathrm{MeV}$. Using the mass of neutron and deuteron as given by Bainbridge ${ }^{2}$, we found the mass of phosphorus-31 to be $30.98375 \pm$ 0.00009 m.u., which agrees, within the experimental errors, with the value of $30.9843 \pm 0.0005$ m.u. obtained by Aston ${ }^{3}$ from the mass-spectroscopic measurements of the mass difference of the CF-P doublet.

Further confirmation is obtained from the work of $\mathrm{Peck}^{4}$ on the reaction ${ }^{30} \mathrm{Si}(d, n)^{31} \mathrm{P}$ and that of Strait, Van Patter, Buechner and Sperduto ${ }^{5}$ on the reaction ${ }^{31} \mathbf{P}(d, \alpha)^{22} \mathrm{Si}$. The $Q$-values of these reactions are $4.56 \pm 0.13 \mathrm{MeV}$. and $8.17 \pm 0.02 \mathrm{MeV}$. respectively. Using the mass of silicon-29 and silicon-30 as reported by Duckworth, Preston and Woodcock ${ }^{8}$, and the mass of the alpha-particle as given by Strait et al. ${ }^{5}$, the mass of phosphorus-31 is found to be $30.98378 \pm 0.00021$ m.u. and $30.98359 \pm 0.00014$ m.u respectively. Both these values are in agreement with our result of $30.98375 \pm 0.00009 \mathrm{~m} . \mathrm{u}$.

Also, from the present determination of the mass of phosphorus-31, the masses of phosphorus-30 and .32 have been calculated from the $Q$-values of the reactions ${ }^{31} \mathrm{P}(\gamma, n)^{30} \mathrm{P}$ and ${ }^{31} \mathrm{P}(d, p)^{32} \mathrm{P}$, as reported by MeElhinney, Hanson, Becker, Duffield and Diven ${ }^{7}$ and by Strait et al..$^{5}$ respectively. The mass of phosphorus-30 is found to be $29.98808 \pm 0.00022 \mathrm{~m} . \mathrm{u}$. and that of phosphorus- 32 is $31.98423 \pm 0.00009$ m.u.

\section{F. A. EL-BEDEWI \\ R. MidDletoN \\ C. T. TAI}

Nuclear Physics Research Laboratory, University of Liverpool. July 31.

Nier, A. O., Phys. Rev., 81, 624 (1951). 2 Bainbridge, K. T., Nat. Res. Coun. Preliminary Report No. 1

${ }^{3}$ Aston, F. W., Proc. Roy. Soc., A, 163, 391 (1937).

4Peck, R. A., Phys. Rev., 73, 947 (1948).

Strait, E. N., Van Patter, D. M., Buechner, W. W., and Sperduto, A., Phys. Rev., 81, 747 (1951).

- Duckworth, H. E., Preston, R. S., and Woodcock, K. S., Phys. Rev., 79, 188 and 402 (1950). ${ }^{2}$ McElhinney, J., Hanson, A. O., Becker, R. A., Duffield, R. B., and
Diven, B. C., Phys. Rev., 75, 542 (1949).

\section{Configuration of the Uranyl lon}

$\mathrm{X}-\mathrm{RAY}$ crystallographic data ${ }^{1,2}$ indicate that in solid uranyl salts and metallic uranates the $\mathrm{UO}_{2}{ }^{++}$ion is linear, and if this configuration is retained, only one frequency corresponding to the symmetric $v_{1}$ vibration should appear in the Raman spectra of uranyl salt solutions. Actual observations ${ }^{3-5}$, however, have shown at least two fundamental frequencies, at about $860 \mathrm{~cm}^{-1}$ and $200 \mathrm{~cm}^{-1}$, which have been assigned to the symmetric $v_{1}$ and bending $v_{2}$ vibrations respectively. Satyanarayana found also a weak line at $909 \mathrm{~cm}^{-1}$, corresponding to the asymmetric $v_{3}$ vibration. These results suggest that the $\mathrm{OO}_{2}++$ ion in solution is bent, a conclusion supported by the infra-red spectral measurements, in which the $v_{1}$ frequency, forbidden for a linear model, has always been observod. Both Crandail ${ }^{5}$ and Satyanarayana have suggested that the ion might be linear in solution, local forces being responsible for the appearance of forbidden frequencies in the spectra. Hitherto, no experimental evidence has been put forward in support of this contention.

Assuming a linear ion, and taking the uranyl fluoride structure as a guide, the uranyl ion in solution is probably hexahydrated, the six water mole. cules forming a puckered ring around the equator of the ion as the $\mathrm{F}-$ ions do in the $\mathrm{UO}_{2} \mathrm{~F}_{2}$ crystal lat.tice ${ }^{2}$. The angle between the $\mathrm{OH}_{2}$ and the $\mathrm{UO}_{2}$ groups will be about $73^{\circ}$. A complexing anion 